Document downloaded from:

http://hdl.handle.net/10251/180694

This paper must be cited as:

Sartiano, D.; Madrigal-Madrigal, J.; Sales Maicas, S. (2019). Sub-cm Temperature Monitoring of 500 Weak Gratings Array Through Chirped Ultra-Short Light Pulses. OSA. 1-2. http://hdl.handle.net/10251/180694

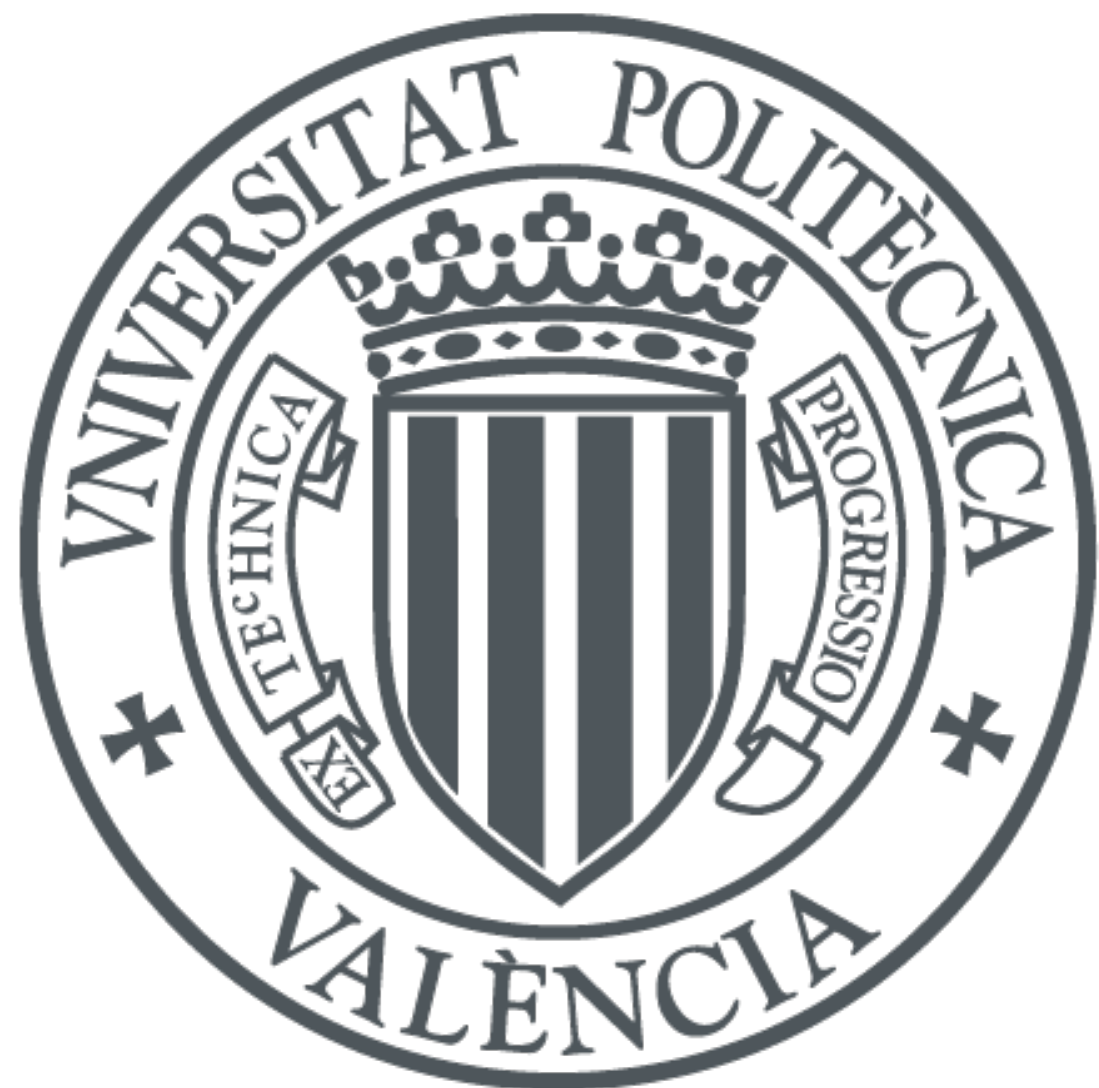

The final publication is available at

https://opg.optica.org/abstract.cfm?uri=sensors-2019-SW5A.5

Copyright OSA

Additional Information

(C) 2019 Optical Society of America]. Users may use, reuse, and build upon the article, or use the article for text or data mining, so long as such uses are for non-commercial purposes and appropriate attribution is maintained. All other rights are reserved. 


\title{
Sub-cm Temperature Monitoring of 500 Weak Gratings Array Through Chirped Ultra-Short Light Pulses
}

\author{
Demetrio Sartiano*, Javier Madrigal, Salvador Sales \\ Institute of Telecommunications and Multimedia Applications (iTEAM), Universitat Politècnica de València, Camino de Vera, s/n 46022 \\ Valencia SPAIN \\ *desar@teleco.upv.es
}

\begin{abstract}
We developed a temperature quasi-distributed sensing system interrogating a 500 weak fiber Bragg gratings (FBGs) array of 5 meters. It was possible to sense temperature changes down to $1^{\circ} \mathrm{C}$ with sub-centimeter spatial resolution.

OCIS codes: (060.2370) Fiber optics sensors; (060.3735) Fiber Bragg gratings; (060.3510) Lasers, fiber.
\end{abstract}

\section{Introduction}

Different techniques were implemented and presented in literature to perform multi-points and quasi-distributed sensing using FBGs arrays and long FBGs: wavelength division multiplexing (WDM) techniques and arrays of FBGs fabricated at different wavelength [1]; interferometry on array of near-identical weak gratings [2]; microwave filtering photonic techniques used to interrogate a weak gratings array [3] and long weak grating [4].

The interrogation methods reported above allow to perform quasi-distributed sensing with some limitation: to use WDM we need to fabricate gratings at different wavelengths and to employ a tunable laser; the microwave filtering techniques need to process the data and to perform a Fourier transform on the frequency spectra acquired, in order to obtain the spatial position of the perturbation applied to the fiber. In fiber sensing the phase sensitive optical time domain reflectometer (OTDR) has lot of advantage to perform distributed sensing, with high spatial resolution and the possibility to interrogate hundreds of kilometers of fiber [5]. This sensing technique allows to sense coherently the Rayleigh backscattered signal in single mode fibers exploiting the OTDR classical setup and light pulses with constant phase. The backscattered signal is quite low power $(<-70 \mathrm{~dB} / \mathrm{m})$, make necessary the employment of very sensitive photodetectors. The idea behind this work is to enhance the back-reflected signal in fiber using an array of 500 weak gratings of $0.9 \mathrm{~cm}$ length and separated of $0.1 \mathrm{~cm}$ and to interrogate it with a short chirped pulse, to obtain high spatial resolution, and use a photodetector with a lower sensitivity than the one required in phase sensitive OTDR. The spatial position of the hot point is calculated on the temporal trace with the time of flight as in OTDR. The chirp was added to the pulse to convert a change in the refractive index in a temporal shift. The laser used is a gain switching laser that generates pulses of less than $10 \mathrm{ps}$ without using an external electro optical modulator (EOM). Even though the gratings are very weak (nominally around $1 \%$ reflectivity), the back-reflected signal is clearly higher than Rayleigh backscattering.

\section{Pulse generation and interrogation setup}

The interrogation technique presented in this work is a wavelength-to-time domain analysis. The interrogation system is implemented with a gain-switching laser diode module [6]. This compact and reliable module uses an attached fiber code whose end is coated with diamond-like carbon (DLC) as a self-seeding pulse feedback circuit (figure 1 left). The laser diode (LD) is biased using a temperature controller. The only external signal needed to active the pulses generation, is a square wave at $5.9 \mathrm{GHz}$, a frequency much lower than the one that should drive an external modulator to generate $10 \mathrm{ps}$ pulses with direct modulation. The setup consists of the laser described above, a dispersion element that introduce -171 $\mathrm{ps} / \mathrm{nm}$ (at $1550 \mathrm{~nm}$ ), and an array of 500 weak FBGs (figure 1 right).
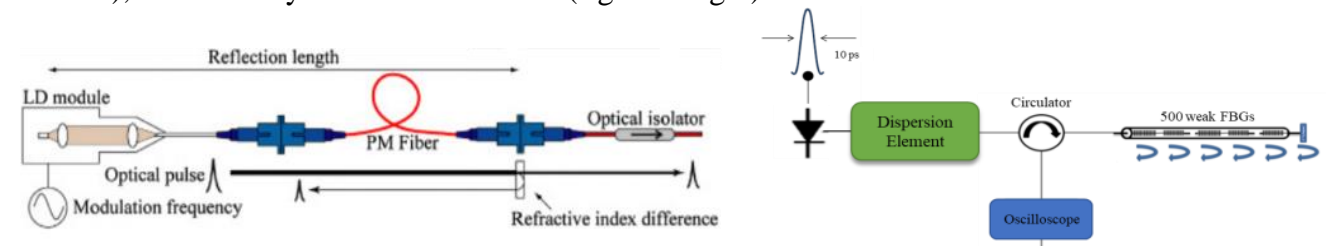

Figure 1. Laser module and optical fiber cavity used for gain-switching implemented with polarization maintaining fiber and two partial reflectors (left). Schematic representation of the experimental setup (right).

The reflected signal was acquired using an oscilloscope triggered on the modulation signal that generate the laser pulses. Hereafter, when a hot zone is provoked along the FBGs array, a temperature gradient is obtained. This local change of temperature will produce a local Bragg frequency shift. The hot point on the array, of approximately $1 \mathrm{~cm}$, was obtained using a temperature closed loop controller implemented with a Peltier and a thermistor. The temperature was swept from $10^{\circ} \mathrm{C}$ to $40^{\circ} \mathrm{C}$ to evaluate the sensitivity and the cumulative error for the measured temperatures. The nominal shift of the FBGs of the array with temperature is of $10 \mathrm{pm} /{ }^{\circ} \mathrm{C}$; to obtain the expected dependence of the temporal shift with 
temperature this value is multiplicated by the dispersion at the laser frequency, obtaining a shift of $1.702 \mathrm{ps} /{ }^{\circ} \mathrm{C}$. The hot point was moved along the gratings array to interrogate two consecutive gratings (spaced of about $1 \mathrm{~cm}$ ).

\section{Results}

In figure $2 \mathrm{a}$ is reported the reflected spectrum of 6 weak gratings around the hot point when the temperature is swept from $10^{\circ} \mathrm{C}$ to $40^{\circ} \mathrm{C}$, with steps of $3^{\circ} \mathrm{C}$. The chirped pulse had lower wavelength at the end of the pulse in time domain; decreasing the temperature the resonant wavelength of the gratings moves to lower wavelength, producing a delay in time of the trace around the hot point. In figure 2 the traces of the reflected signal when the temperature sweep is applied in one point (a) and moved to another grating of the array (b). The resolution given by the pulse width allows to clearly distinguish the two cases. With this system was possible not only to sense the temperature in the hot point but as well to observe the temperature gradient along it, observed as a gradually decrease of the time shift when we move far from the hot point. In order to evaluate the linearity of the system with the temperature a threshold was fixed to a certain temperature and the time stamp of consecutive traces was derived. The result is showed in figure $2 \mathrm{c}$. The absolute time stamps of the traces are linear with temperature $(R=0.99389)$. The mean value and the standard deviation for the measured time shifts were $1.9 \pm 0.3505 \mathrm{ps}$, close to the expected value of $1.7 \mathrm{ps}$. Still the standard deviation is quite high due to the cumulative error of the measurement. This limitation and the need of different algorithms for processing, were addressed in phase sensitive OTDR application with chirped pulse as well [5].

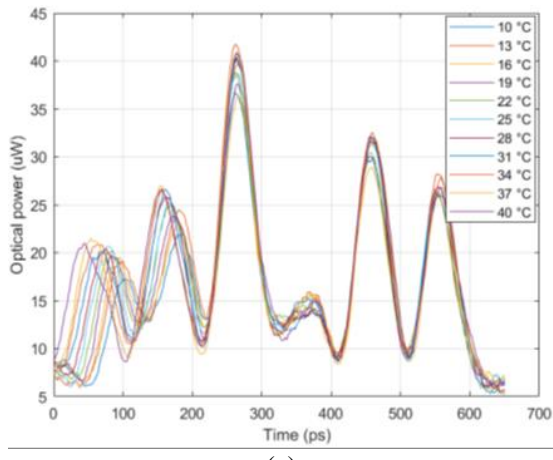

(a)

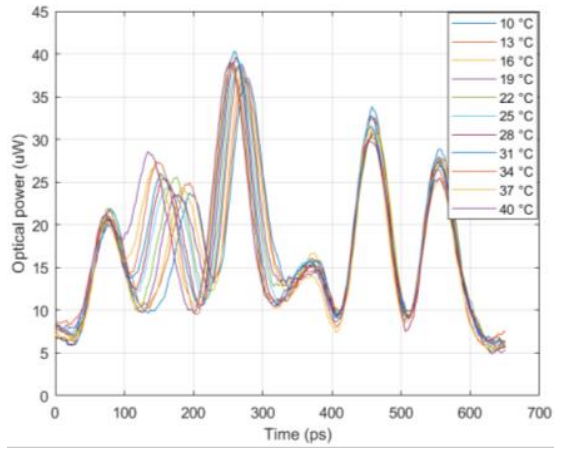

(b)

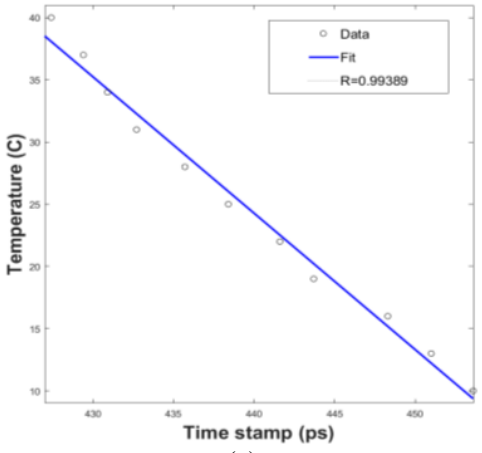

(c)

Figure 2. (a), (b) Reflected signal of the array section under test. The two images present the heating of two point spaced of less than one centimeter. In both cases is visible the time shift due to the increasing temperature from $10^{\circ} \mathrm{C}$ to $40^{\circ} \mathrm{C}$. (c) Evaluation of the response of our sensor to temperature: when the temperature was swept from $10^{\circ} \mathrm{C}$ to $40^{\circ} \mathrm{C}$ we can observe a linear dependence with the time stamp of the traces

\section{Conclusion and future works}

We presented a quasi-distributed sensing system of a long weak gratings array of 5 meters, using a compact pulsed laser module without the employment of external EOM. The chirp introduced in the interrogation pulse allows to convert any refractive index changes inside the 500 weak grating arrays in a time shift. It was possible to interrogate a long array of FBGs, fabricated at the same wavelength, independently. The weak gratings reflect part of the signal and allows to use an optical oscilloscope to recover the reflected traces. The sensitivity of our system was limited to $1^{\circ} \mathrm{C}$, due to the maximum sampling rate (2 ps) of the oscilloscope. Applying a higher dispersion to the pulse and using a detection system with higher sampling rate is possible to enhance the sensitivity. New processing algorithms and a deep understanding of the effect of the cumulative error, and how to minimize its effect, will be addressed in future works.

\section{Funding}

This research was supported by FINESSE project, funded by the European Union's Horizon 2020 research and innovation program under the Marie Sklodowska-Curie Action grant agreement $\mathrm{n}^{\circ} 722509$. It was also supported by the Ministry of Economy and Competitiveness project DIMENSION TEC2017 and by the Generalitat Valenciana project PROMETEO $2017 / 017$.

\section{References}

[1] Chan, Peter KC, et al. "Multiplexing of fiber Bragg grating sensors using a FMCW technique." IEEE Photonics Technology Letters 11.11 (1999): 1470-1472.

[2] Ou, Yiwen, et al. "Large-capacity multiplexing of near-identical weak fiber Bragg gratings using frequency-shifted interferometry." Optics express 23.24 (2015): 31484-31495.

[3] Hervás, Javier, et al. "Microwave Photonics filtering interrogation technique under coherent regime for hot spot detection on a weak FBGs array." Journal of Lightwave Technology 36.4 (2018): 1039-1045.

[4] Ricchiuti, Amelia L., et al. "Cascade FBGs distributed sensors interrogation using microwave photonics filtering techniques." Optics \& Laser Technology 77 (2016): 144-150.

[5] Pastor-Graells, Juan, et al. "Single-shot distributed temperature and strain tracking using direct detection phase-sensitive OTDR with chirped pulses." Optics express 24.12 (2016): 13121-13133.

[6] Nonaka, Koji, et al. "Low-time-jitter short-pulse generator using compact gain-switching laser diode module with optical feedback fiber line." Japanese Journal of Applied Physics 47.8S1 (2008): 6754. 